\title{
Universiteit
}

Leiden

The Netherlands

\section{Direct measurement of transverse-mode entanglement in two-photon states}

Lorenzo Pires, H. di; Monken, C.H.; Exter, M.P. van

\section{Citation}

Lorenzo Pires, H. di, Monken, C. H., \& Exter, M. P. van. (2009). Direct measurement of transverse-mode entanglement in two-photon states. Physical Review A, 80, 022307. doi:10.1103/PhysRevA.80.022307

Version: $\quad$ Not Applicable (or Unknown)

License: $\quad$ Leiden University Non-exclusive license

Downloaded from: https://hdl.handle.net/1887/61255

Note: To cite this publication please use the final published version (if applicable). 


\title{
Direct measurement of transverse-mode entanglement in two-photon states
}

\author{
H. Di Lorenzo Pires, ${ }^{1}$ C. H. Monken, ${ }^{1,2}$ and M. P. van Exter ${ }^{1}$ \\ ${ }^{1}$ Huygens Laboratory, Leiden University, P.O. Box 9504, 2300 RA Leiden, The Netherlands \\ ${ }^{2}$ Departamento de Física, Universidade Federal de Minas Gerais, Caixa Postal 702, Belo Horizonte, MG 30123-970, Brazil
}

(Received 6 March 2009; published 5 August 2009)

\begin{abstract}
We introduce and experimentally demonstrate a method to measure the Schmidt number of pure two-photon states entangled in transverse-mode structure. Our method is based on the connection between the Schmidt decomposition in quantum theory and the coherent-mode decomposition in classical coherence theory. We apply the method to two-photon states generated by spontaneous parametric down conversion and show that our results are in excellent agreement with numerical calculations based on the Schmidt decomposition.
\end{abstract}

DOI: 10.1103/PhysRevA.80.022307

PACS number(s): 03.67.Bg, 03.67.Mn, 42.50.Dv, 42.65.Lm

Entanglement is an old concept in quantum mechanics that has only recently been recognized as a key resource in quantum information science. In order to take full advantage of the capabilities of a given quantum system for quantum information processing, it is crucial to thoroughly characterize its entanglement and to measure the amount of this resource that is available, a measurement that goes beyond the detection of entanglement signatures.

In the last years, there has been a significant effort to design and implement strategies to measure entanglement without prior state reconstruction [1]. With the increasing dimension of the system, techniques such as quantum state tomography become more and more involved and should, therefore, be avoided. Most of the progress so far has been achieved for simple two-qubit systems. For instance, in Ref. [2]. Walborn et al. reported the first experimental measurement of concurrence for pure two-qubit states. An estimation of the concurrence for mixed states was also realized [3]. Furthermore, many proposals to experimentally quantify the entanglement in low-dimensional bipartite systems are being introduced [4-8].

Though the majority of the analyses focuses on realizations with two qubits, there is a growing interest in the highdimensional entanglement of continuous-variable systems. In fact, many existing quantum processing protocols could be boosted by employing larger alphabets [9]. For these intrinsically more complex spaces, the question of whether the amount of entanglement can be obtained without a full state tomography becomes particularly appealing.

The most convenient parameter to quantify the amount of entanglement in a continuous-variable pure bipartite state $|\Psi\rangle$ is the Schmidt number $K$, defined as the "average" or "effective" number of nonzero coefficients in the Schmidt decomposition [10]

$$
|\Psi\rangle=\sum_{n=0}^{\infty}{\sqrt{\lambda_{n}}}_{n}\left|\phi_{n}\right\rangle \otimes\left|\psi_{n}\right\rangle .
$$

Equivalently, the Schmidt number $K$ is the inverse of the purity of the reduced density operator $\varrho_{1}$ (or $\varrho_{2}$ )

$$
\varrho_{1}=\operatorname{Tr}_{2}[|\Psi\rangle\langle\Psi|]=\sum_{n=0}^{\infty} \lambda_{n}\left|\phi_{n}\right\rangle\left\langle\phi_{n}\right| .
$$

Thus,

$$
K=\frac{\left(\sum_{n=0}^{\infty} \lambda_{n}\right)^{2}}{\sum_{n=0}^{\infty} \lambda_{n}^{2}}=\frac{\left(\operatorname{Tr} \varrho_{1}\right)^{2}}{\operatorname{Tr} \varrho_{1}^{2}}=\frac{\left(\operatorname{Tr} \varrho_{2}\right)^{2}}{\operatorname{Tr} \varrho_{2}^{2}} .
$$

Other appropriate quantifiers, such as the $I$ concurrence [11], can be written in terms of the Schmidt number. For global pure states, each part carries all the information about the amount of entanglement present, as one can conclude by comparing Eqs. (1) and (2). The more entangled are the parts, the more mixed are the reduced states. The above definition, though mathematically simple, does not provide a clear way for the experimental measurement of the Schmidt number [12]. Due to the high number of terms involved in Eq. (1), even a numerical calculation of $K$ may be difficult and time demanding. In view of these difficulties, "experimentally friendly" parameters were introduced, such as the ratio of widths of single-particle and coincidence distributions [13], in an attempt to directly quantify entanglement.

In this work we show that it is possible to give an operational meaning to the Schmidt number in the framework of coherence theory and present a method to evaluate and measure, with the least experimental effort, the amount of entanglement associated with the spatial degrees of freedom of an entangled photon pair. For this method to work, we only need to assume that the global state is pure and the reduced states have a sufficiently homogeneous statistics.

In photonic states, the notion of "mixedness" is related to coherence. In fact, the transverse coordinate $(\mathbf{x})$ representation of the reduced density operator in Eq. (2) is proportional to the cross-spectral density function of the source $W_{s}\left(\mathbf{x}, \mathbf{x}^{\prime}\right) \propto\left\langle\mathbf{x}\left|\varrho_{1}\right| \mathbf{x}^{\prime}\right\rangle$, introduced and analyzed by Wolf [14]. The fields are assumed to be monochromatic. A more appealing, operationally defined function, is the cross-spectral degree of coherence, obtained by normalizing $W_{s}$ by the intensities, i.e., $\mu_{s}\left(\mathbf{x}, \mathbf{x}^{\prime}\right)=W_{s}\left(\mathbf{x}, \mathbf{x}^{\prime}\right) / \sqrt{I_{s}(\mathbf{x}) I_{s}\left(\mathbf{x}^{\prime}\right)}$, where $I(\mathbf{x})$ $=W_{s}(\mathbf{x}, \mathbf{x})$. If the considered two-photon state is entangled, the reduced one-photon state can never be fully coherent. The more entangled the two-photon state is, the more incoherent is each one of its one-photon components. The average transverse spectral degree of coherence of the onephoton states can be quantified by the overall degree of coherence [15], defined by 


$$
\bar{\mu}^{2}=\frac{\iint\left|W_{s}\left(\mathbf{x}, \mathbf{x}^{\prime}\right)\right|^{2} d \mathbf{x} d \mathbf{x}^{\prime}}{\left[\int\left|W_{s}(\mathbf{x}, \mathbf{x})\right| d \mathbf{x}\right]^{2}} .
$$

It can also be seen as an average of the cross-spectral degree of coherence as $\bar{\mu}^{2}=\iint P\left(\mathbf{x}, \mathbf{x}^{\prime}\right)\left|\mu_{s}\left(\mathbf{x}, \mathbf{x}^{\prime}\right)\right|^{2} d \mathbf{x} d \mathbf{x}^{\prime}$, where $P\left(\mathbf{x}, \mathbf{x}^{\prime}\right)=I_{s}(\mathbf{x}) I_{s}\left(\mathbf{x}^{\prime}\right) /\left[\int I_{s}(\mathbf{x}) d \mathbf{x}\right]^{2}$.

It is well known from the classical theory of coherence that the cross-spectral density function admits a coherentmode representation [14],

$$
W_{s}\left(\mathbf{x}, \mathbf{x}^{\prime}\right)=\sum_{n=0}^{\infty} c_{n} \phi_{n}^{*}(\mathbf{x}) \phi_{n}\left(\mathbf{x}^{\prime}\right),
$$

where the functions $\phi_{n}$ form an orthonormal set and the coefficients $c_{n}$ are all positive. Although derived in different contexts, Eqs. (2) and (5) refer to the same physical property of the source. Therefore, using Eqs. (2), (4), and (5) one can see that the Schmidt number $K$ is

$$
K=\frac{1}{\bar{\mu}^{2}} \text {. }
$$

This is our first key result. We present another physical meaning to the Schmidt number of two-photon entangled states: it is the inverse of the overall degree of coherence of the reduced state. Although Eq. (6) is always valid and provides a path to the direct measurement of $K$, the experimental determination of $\bar{\mu}^{2}$ may require an enormous experimental effort. However, in some important practical situations this effort is considerably reduced, as we will now show.

Depending on the characteristics of the two-photon source, especially if it is highly entangled, its reduced (onephoton) state may describe a quasihomogeneous source. For this class of sources [14], the cross-spectral degree of coherence $\mu_{s}\left(\mathbf{x}, \mathbf{x}^{\prime}\right)$ depends locally only on the difference $\mathbf{x}-\mathbf{x}^{\prime}$ and decays to zero if $\left|\mathbf{x}-\mathbf{x}^{\prime}\right|$ is greater than the transverse coherence length of the source, within which the positional intensity profile $I_{s}(\mathbf{x})$ is smoothly varying, that is, $I(\mathbf{x}) I\left(\mathbf{x}^{\prime}\right)$ $\approx I^{2}\left[\frac{1}{2}\left(\mathbf{x}+\mathbf{x}^{\prime}\right)\right]$. In this case, the cross-spectral density function may be approximated by $W_{s}\left(\mathbf{x}, \mathbf{x}^{\prime}\right) \approx I\left[\frac{1}{2}\left(\mathbf{x}+\mathbf{x}^{\prime}\right)\right] g_{s}(\mathbf{x}$ $\left.-\mathbf{x}^{\prime}\right)$. This factorization is exact for Gaussian sources. Defining $\widetilde{g}_{s}(\mathbf{q})$ as the Fourier transform of $g_{s}(\mathbf{x})$, it can be shown $[14]$ that $\widetilde{g}_{s}(\mathbf{q})$ is proportional to the far-field intensity $I_{F F}(\boldsymbol{\theta})$, where $\boldsymbol{\theta}=\mathbf{q} / k, k$ being the wave number. Using the facts just mentioned, and Eqs. (4) and (6), the Schmidt number can be written as

$$
K \approx \frac{1}{\lambda^{2}} \frac{\left[\int I_{S}(\mathbf{x}) d \mathbf{x}\right]^{2}}{\int I_{s}^{2}(\mathbf{x}) d \mathbf{x}} \times \frac{\left[\int I_{F F}(\boldsymbol{\theta}) d \boldsymbol{\theta}\right]^{2}}{\int I_{F F}^{2}(\boldsymbol{\theta}) d \boldsymbol{\theta}},
$$

where $I_{s}$ and $I_{F F}$ are the intensities measured on the source (near field) and on the far field, respectively, and $\lambda$ is the wavelength. We see that, with two intensity profile measurements (in near and far fields), it is possible to obtain the Schmidt number $K$ directly, without the need of a full state tomography. This is our second key result, which forms the basis of our experimental analysis.

We now illustrate the use of the proposed method to estimate the Schmidt number associated with the transversemode entanglement in the two-photon field generated by spontaneous parametric down-conversion (SPDC) in a periodically poled crystal. The two-photon state generated by quasimonochromatic type I SPDC is pure and its wave function in momentum representation assumes the form [16]

$$
\Phi\left(\mathbf{q}_{1}, \mathbf{q}_{2}\right)=\mathcal{N} \mathcal{E}_{p}\left(\mathbf{q}_{1}+\mathbf{q}_{2}\right) \operatorname{sinc}\left(b^{2}\left|\mathbf{q}_{1}-\mathbf{q}_{2}\right|^{2}+\varphi\right),
$$

where $\mathcal{N}$ is a normalization constant, $\mathcal{E}_{p}(\mathbf{q})$ is the plane-wave spectrum of the pump beam, assumed to have the Gaussian profile $\exp \left(-|\mathbf{q}|^{2} / \sigma^{2}\right)$. The sinc function arises from phase matching, $b^{2}=L / 4 n k_{p}, L$ is the crystal thickness, $k_{p}$ is the wave number of the pump beam, $n$ is the refractive index for the down-converted field, and $\varphi$ is the collinear phase mismatch parameter. The adequacy of Eq. (8) to represent the two-photon state generated by type I SPDC has been confirmed in a number of published works. In particular, the assumption about its purity in the quasimonochromatic regime (when narrow band frequency filters are used) is supported by the high visibilities exhibited in fourth-order interference experiments in a wide range of conditions [17-19]. In order to compare with the results published in Ref. [10], we use the notation of Law and Eberly, where $w_{p}=2 / \sigma$ is the width of the Gaussian pump beam. It is not difficult to show that if $b \sigma \ll 1$ the SPDC reduced density matrix $\left\langle\mathbf{q}^{\prime}\left|\varrho_{1}\right| \mathbf{q}\right\rangle$ $=\int \Phi^{*}\left(\mathbf{q}^{\prime}, \mathbf{q}_{2}\right) \Phi\left(\mathbf{q}, \mathbf{q}_{2}\right) d \mathbf{q}_{2}$ leads to the factorized expression for $\bar{\mu}^{2}$ characteristic of a quasihomogeneous source, so that Eq. (7) holds.

The predictions of Eq. (7) are confirmed by a numerical calculation of the Schmidt number for SPDC based on the Schmidt decomposition of the two-photon state described by Eq. (8), that is, $\Phi\left(\mathbf{q}_{1}, \mathbf{q}_{2}\right)=\Sigma_{n} \sqrt{\lambda_{n}} u_{n}\left(\mathbf{q}_{1}\right) v_{n}\left(\mathbf{q}_{2}\right)$. One can show that $K$ depends only on two parameters: the product $b \sigma$ and the phase mismatch $\varphi$. We follow [10] and obtain $K$ for different values of $b \sigma$. In addition, we investigate for the first time how the entanglement depends on the phase mismatch parameter $\varphi$. This calculation is lengthy and requires some computational power. Alternatively, we use Eq. (7) and immediately obtain a good approximation for the Schmidt number.

In Fig. 1 we compare the exact and approximated results. In Fig. 1(a) we keep $\varphi=0$ and vary $b \sigma$ from 0.05 to 0.5 . The smaller the value of $b \sigma$, the more the two-photon field approximates the maximally entangled state $\delta\left(\mathbf{x}_{1}-\mathbf{x}_{2}\right)$ and the higher is the Schmidt number. It is known that changes in the phase mismatch parameter $\varphi$ lead to dramatic effects in the far-field intensities without any change in the near field. The Schmidt number predicted by Eq. (7) should change accordingly. In Fig. 1(b) we compare the exact and approximated results for $K$ calculated at a fixed $b \sigma=0.1$ and for a wide range of the phase mismatch parameter $\varphi$. We conclude that Eq. (7) indeed provides a very good approximation to $K$, especially in the regime $b \sigma \ll 1$.

Next, we demonstrate that under the conditions assumed (pure two-photon state and quasihomogeneous reduced onephoton state) the Schmidt number can be measured in a 

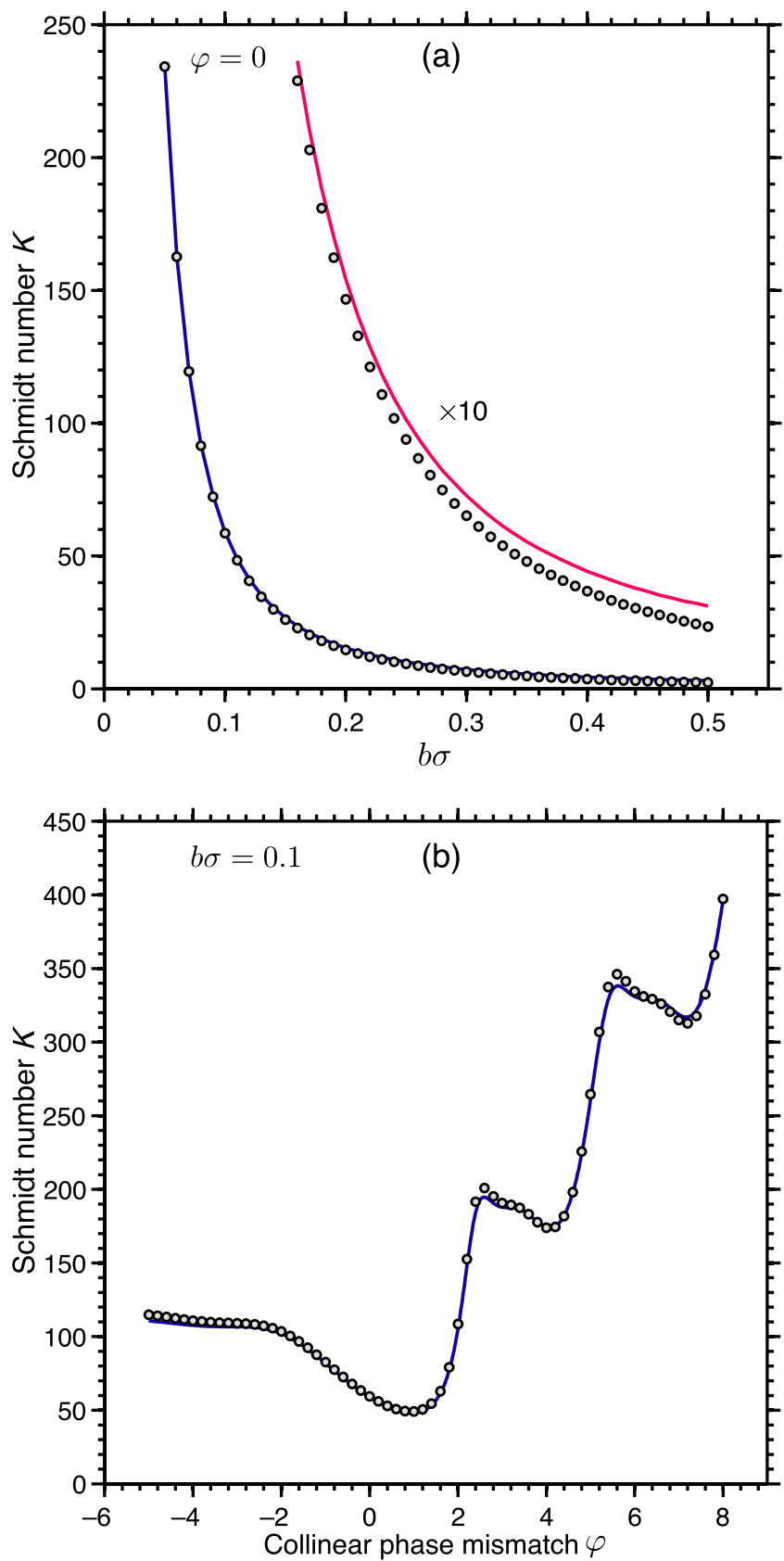

FIG. 1. (Color online) Schmidt number $K$ obtained from Eq. (7) (solid line) and calculated via mode decomposition (circles). The top graph (a) shows $K$ as a function of $b \sigma$ for $\varphi=0$. The $\times 10$ magnified curve shows that the approximation of Eq. (7) is correct to within a few percent even for $K$ as low as 10 . The bottom graph (b) shows $K$ as a function of $\varphi$ for $b \sigma=0.1$.

simple experiment. For this purpose we use the setup depicted in Fig. 2. Spatially entangled photon pairs are produced by type I SPDC in a $5.06 \mathrm{~mm}$-thick periodically poled $\mathrm{KTiOPO}_{4}$ crystal (PPKTP) pumped by a mildly focused Krypton laser beam $\left(\lambda=413 \mathrm{~nm}, w_{p}=162 \mu \mathrm{m}\right)$. After the crystal, the laser light is blocked by a filter $\left(F_{1}\right)$ and the intensity profile of the down-converted light is measured with an intensified charge coupled device (ICCD) camera. A spectral filter $\left(F_{2}\right)$ is used to select the degenerate frequency component. The detection bandwidth $(5 \mathrm{~nm}$ at $826 \mathrm{~nm})$ is

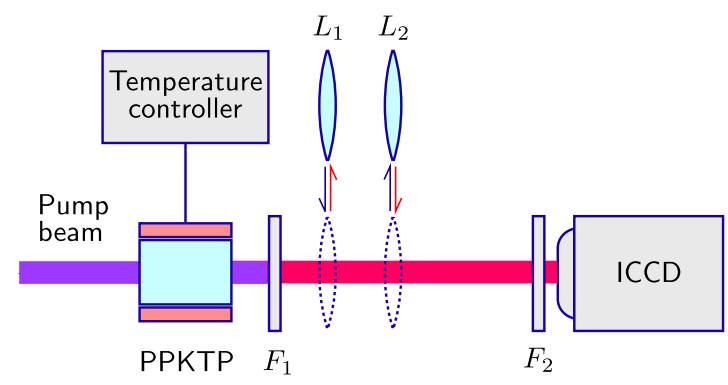

FIG. 2. (Color online) Experimental setup comprising a pumped crystal (PPKTP) and optics to create images of either the near field or the far-field profile onto an ICCD.

small enough to limit spatial-spectral correlations to an undetectable level (mismatch parameter $\varphi$ varies $<0.1$ over this bandwidth [20]). Since our camera is not sensitive to photon correlations, there is no need to split the photon pairs. To measure the near field intensity, a $12 \times$ magnified image of the transverse plane at the center of the crystal is created on the detection area with a $59 \mathrm{~mm}$ focal-length lens $\left(L_{1}\right)$. For the far-field intensity, a $f-f$ configuration is set up with a 100 $\mathrm{mm}$ focal-length lens $\left(L_{2}\right)$. The phase mismatch parameter $\varphi$ can be adjusted by changing the temperature of the crystal. Based on the temperature dependence of the refractive indices at the pump and SPDC wavelengths, the derivative $d \varphi / d T \approx 1.04 \mathrm{~K}^{-1}$ around the collinear phase matching temperature $T_{0} \approx 60{ }^{\circ} \mathrm{C}$ was calculated and checked experimentally [20]. To subtract the background noise, we record, along with each measurement, a background image, taken when the polarization of the pump is rotated by $90^{\circ}$, suppressing down conversion. The intensities in the near field and far field are measured for many different values of $\varphi$. After subtracting the background, we use Eq. (7) to estimate the Schmidt number of the two-photon state.

The key experimental result of this work is depicted in Fig. 3. The circles represent the Schmidt number $K$ experimentally obtained. The solid line is the theoretical prediction based on Eqs. (7) and (8) with $b \sigma=0.077$. This value for $b \sigma$ was obtained by curve fitting the near- and far-field intensity profiles for $\varphi=0$. We observe that there is a good qualitative and quantitative agreement between theory and experiment. Even the peculiar details of the curve are reproduced experimentally. Some examples of how the angular emission pattern changes with $\varphi$ are also shown. For larger values of $\varphi$, as the effective width of the transverse structure of the downconverted fields rapidly increases, the finite extent of the detection area becomes important. This finite detection area sets practical limits to the integration domains in Eq. (7), leading to a reduction of the detected values of $K$. With this correction taken into account, the theoretical prediction for the detected Schmidt number is represented by the dashed line. It should be noticed that while $K$ may achieve very high values for $\varphi>0$, the down-conversion efficiency drops significantly, due to the lack of phase matching. More precisely, the overall photon flux $F(\varphi)$ relative to its $\varphi=0$ value is given by $F(\varphi) / F(0)=1+(2 / \pi)\left[\varphi \operatorname{sinc}^{2} \varphi-\operatorname{Si}(2 \varphi)\right]$, where $\operatorname{Si}(x)=\int_{0}^{x} \operatorname{sinc} x^{\prime} d x^{\prime}$ is the sine integral function. For example, $F(4) / F(0) \approx 0.09$ and $F(8) / F(0) \approx 0.04$.

The results in Fig. 3 can be qualitatively explained as 


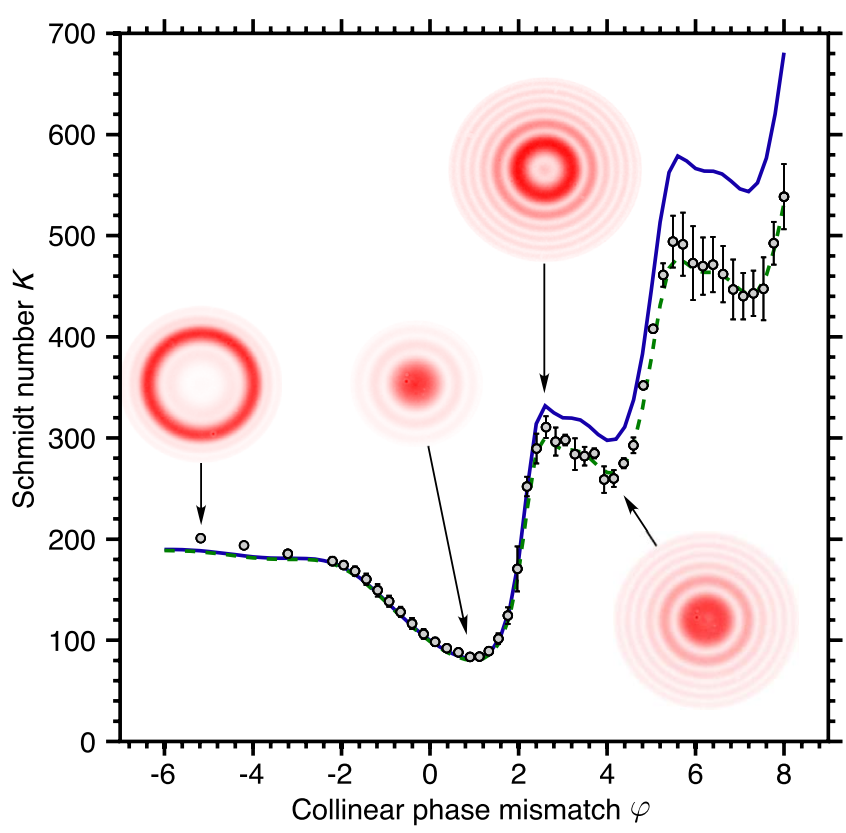

FIG. 3. (Color online) Experimental results (circles), theoretical prediction for infinite detection area (solid line), and theoretical prediction corrected for the finite size of the detection area (dashed line). Some of the measured far-field intensity profiles are shown.

follows. For negative phase mismatch $(\varphi<0)$ the amount of entanglement does not depend strongly on $\varphi$. This is because the SPDC far field is concentrated in a "ring" whose area is practically independent of the radius. Around $\varphi=0$, the ring collapses into a central spot of smaller area, reducing the ratio in Eq. (7). At $\varphi \approx 1$ the central peak resembles a Gaussian and $K$ reaches its minimum value. For $\varphi>1$, the main ring completely disappears, and the weaker, secondary peaks of the sinc function lead to a more spread intensity distribu- tion, rapidly increasing the value of $K$. As $\varphi$ increases, the effective width of the far-field intensity profile keeps increasing, oscillating with $\varphi$ due to rearrangements in the rings structure, thus leading to oscillations in the value of $K$.

Finally, we interpret the product of the ratios in Eq. (7) taken at the near and far field, as the product of an effective "object" area $A_{\text {eff }}$ (near field) and an effective emission angle $\Omega_{\text {eff }}$ (far field) via

$$
K=\frac{1}{\lambda^{2}} A_{e f f} \Omega_{e f f} .
$$

The same formula is known in classical optics as the optical étendue or the Shannon number of an image or imaging system. The product $A_{e f f} \Omega_{e f f} / \lambda^{2}$ defines the number of independent classical communication channels available to the optical system. We provide, in this way, support to the recently proposed relation between two-photon spatial entanglement and the Shannon dimensionality of quantum channels [21]. Needless to say, this association is valid for the conditions assumed here: pure two-photon states with quasihomogeneous reduced one-photon states.

In conclusion, we reported a measurement of entanglement in an infinite-dimensional space. We proved that the Schmidt number of the transverse-mode entanglement of a two-photon field is identical to the inverse of the overall degree of coherence of the source. The theoretical framework based on the coherence theory indicates, contrary to what is usually assumed, that the amount of spatial entanglement can be experimentally estimated in a straightforward way.

This work has been supported by the Stichting voor Fundamenteel Onderzoek der Materie (FOM). C.H.M. acknowledges support from the Brazilian agencies $\mathrm{CNPq}$ and CAPES.
[1] P. Horodecki, Phys. Rev. Lett. 90, 167901 (2003).

[2] S. P. Walborn et al., Nature (London) 440, 1022 (2006).

[3] C. Schmid, N. Kiesel, W. Wieczorek, H. Weinfurter, F. Mintert, and A. Buchleitner, Phys. Rev. Lett. 101, 260505 (2008).

[4] F. Mintert and A. Buchleitner, Phys. Rev. Lett. 98, 140505 (2007).

[5] J. Cai and W. Song, Phys. Rev. Lett. 101, 190503 (2008).

[6] S. M. Lee, S. W. Ji, H. W. Lee, and M. Suhail Zubairy, Phys. Rev. A 77, 040301(R) (2008).

[7] L. Aolita, A. Buchleitner, and F. Mintert, Phys. Rev. A 78, 022308 (2008).

[8] L. Neves, G. Lima, E. J. S. Fonseca, L. Davidovich, and S. Padua, Phys. Rev. A 76, 032314 (2007).

[9] H. Bechmann-Pasquinucci and W. Tittel, Phys. Rev. A 61, 062308 (2000).

[10] C. K. Law and J. H. Eberly, Phys. Rev. Lett. 92, 127903 (2004).

[11] P. Rungta, V. Buzek, C. M. Caves, M. Hillery, and G. J. Milburn, Phys. Rev. A 64, 042315 (2001).

[12] M. V. Fedorov, M. A. Efremov, P. A. Volkov, E. V. Moreva, S.
S. Straupe, and S. P. Kulik, Phys. Rev. A 77, 032336 (2008).

[13] M. V. Fedorov, M. A. Efremov, A. E. Kazakov, K. W. Chan, C. K. Law, and J. H. Eberly, Phys. Rev. A 69, 052117 (2004).

[14] E. Wolf, Introduction to the Theory of Coherence and Polarization of Light (Cambridge University Press, New York, 2007).

[15] M. J. Bastiaans, Opt. Acta 28, 1215 (1981).

[16] C. H. Monken, P. H. Souto Ribeiro, and S. Pádua, Phys. Rev. A 57, 3123 (1998).

[17] T. Yarnall, A. F. Abouraddy, B. E. A. Saleh, and M. C. Teich, Phys. Rev. Lett. 99, 170408 (2007).

[18] W. H. Peeters and M. P. van Exter, Phys. Rev. A 79, 043817 (2009).

[19] H. Di Lorenzo Pires and M. P. van Exter, Phys. Rev. A 79, 041801(R) (2009).

[20] W. H. Peeters and M. P. van Exter, Opt. Express 16, 7344 (2008).

[21] J. B. Pors, S. S. R. Oemrawsingh, A. Aiello, M. P. van Exter, E. R. Eliel, G. W. t Hooft, and J. P. Woerdman, Phys. Rev. Lett. 101, 120502 (2008). 